Published in final edited form as:

J Card Fail. 2016 December ; 22(12): 996-1003. doi:10.1016/j.cardfail.2016.10.008.

\title{
Transthyretin Cardiac Amyloidosis in Older Americans
}

\author{
Danielle L. Brunjes, Adam Castano, Autumn Clemons, Jonah Rubin, and Mathew S. \\ Maurer $^{*}$ \\ Division of Cardiology, Department of Medicine, Columbia University Medical Center, New York, \\ NY
}

\section{Abstract}

Wild type transthyretin cardiac amyloidosis (ATTRwt), formerly called senile cardiac amyloidosis (SCA), is almost exclusively a disorder of older adults. As the population ages, the diagnosis of ATTRwt will increase making it the most common form of cardiac amyloidosis. An important precondition to reduce under-diagnosis and misdiagnosis is to maintain a high index of suspicion for cardiac amyloidosis. Several clues can be gleaned from the clinical history, physical exam, electrocardiogram and non-invasive imaging techniques. Nuclear scintigraphy agents using ${ }^{99 \mathrm{~m}} \mathrm{Tc}-$ phosphate derivatives combined with assessment for monoclonal proteins are eliminating the need for tissue confirmation in ATTR cardiac amyloid. Morbidity and mortality from ATTRwt cardiac amyloid is high and the emergence of numerous therapies based on a biologic understanding of the pathophysiology of this condition including drugs to inhibit the synthesis of TTR, stabilize TTR, and degrade or extract amyloid provides new hope for those afflicted. This review will briefly cover the epidemiology, pathophysiology and clinical manifestations, as well as diagnostic strategies and treatment of older adults with ATTR cardiac amyloid.

\section{Keywords}

Transthyretin cardiac amyloidosis; geriatric heart failure; diagnostic strategies

\section{INTRODUCTION}

\begin{abstract}
Amyloidosis is caused by unstable protein structures that misfold, aggregate and form amyloid fibrils leading to disruptions in organ function.[1] Two distinct types of transthyretin (TTR) amyloidosis that are known to have a predilection for the heart in patients of advanced age include wild type (ATTRwt), previously known as senile cardiac amyloidosis (SCA), $[1,2]$ and hereditary or mutant (ATTRm). Both cause restrictive cardiomyopathy, with thickened and stiffened ventricles that result in severe diastolic dysfunction.[1-5] ATTRwt cardiac amyloidosis is almost exclusively a disorder of adults
\end{abstract}

\footnotetext{
*Address for correspondence: Mathew S. Maurer, MD, Clinical Cardiovascular Research Lab for the Elderly, Columbia University Medical Center, Department of Medicine, Division of Cardiology, 622 West 168th Street, PH 12 Room 134, New York, NY 10032, USA, Tel: 212-305-9808 Fax: 212-305-7439, msm10@cumc.columbia.edu.

Publisher's Disclaimer: This is a PDF file of an unedited manuscript that has been accepted for publication. As a service to our customers we are providing this early version of the manuscript. The manuscript will undergo copyediting, typesetting, and review of the resulting proof before it is published in its final citable form. Please note that during the production process errors may be discovered which could affect the content, and all legal disclaimers that apply to the journal pertain.
} 
over the age of 60 and represents a quintessential type of diastolic heart failure in the geriatric population.[6] This brief review focuses on increasing recognition of ATTRwt and ATTRm in older adults, including features that should elevate clinical suspicion for the condition, non-biopsy diagnosis and emerging treatment strategies.

\section{EPIDEMIOLOGY}

According to the US Census Bureau, the percentage of the population $>65$ years was $\sim 12.4 \%$ in 2000 and is currently $>26 \%$. [7] Systemic light chain (AL) amyloidosis has been considered the most common form of systemic amyloidosis, but the diagnosis of ATTRwt and ATTRm cardiac amyloidosis are increasing because of the aging population and emergence of noninvasive diagnostic imaging technologies.[8] The most common mutation in the United States, the valine-to-isoleucine mutation at position 122 (Val122Ile), has a prevalence of $4 \%$ in African Americans. [9] Cardiac involvement in ATTRwt was often a postmortem finding, considered to be a normal part of the aging process.[4] Using immunohistochemistry to identify the precursor protein, the prevalence of ATTRwt on autopsies in subjects $\geq 80$ years old has been reported to be approximately $25 \%$.[10] More recently, 32\% of subjects with heart failure and preserved ejection fraction (HFpEF) $\geq 75$ years old had TTR cardiac amyloid on autopsy, of which only $21 \%$ had a pre-morbid diagnosis of amyloid.[11] Multiple series of patients presenting to referral centers have found ATTRwt and ATTRm cardiac amyloidosis to be predominately disorders of older adult males.[6, 12, 13] However, using technetium scintigraphy to actively screen and identify subjects with TTR cardiac amyloidosis, $13 \%$ of subjects hospitalized with HFpEF and wall thicknesses $\geq 12 \mathrm{~mm}$ had ATTRwt cardiac amyloid, notably without gender differences, suggesting that both older adult men and women may be underdiagnosed.[14, 15]

The Transthyretin Amyloidosis Outcomes Survey (THAOS), the largest ongoing, global, multicenter, longitudinal, observational study has characterized differences in the disease course and outcomes in geographically dispersed patients with ATTR cardiac amyloidosis. In the United States, ATTRwt accounted for $48 \%$ of the ATTR cases, followed by the Val122Ile mutation which accounted for $23 \%$. Of note, $85 \%$ of the Val122Ile patients in THAOS were from the United States. Outside of the United States, only 5\% of patients had ATTRwt and 76\% had ATTRm due to the Val30Met mutation.[16] Compared with ATTRm due to the Val122Ile mutation, subjects with ATTRwt were older (76 \pm 7 vs. $69 \pm 10$ years) and almost exclusively male (97.4\% vs. $75.8 \%$ ). ATTRwt patients were mostly Caucasian $(89.4 \%)$ while ATTRm Val122Ile patients were mostly of African descent (86.8\%). These multicenter data confirm the marked racial differences that exist between those with ATTRwt cardiac amyloidosis and those with the Val122Ile mutation.[1, 11, 16]

\section{PATHOPHYSIOLOGY}

The TTR protein is a 127 amino acid structure rich in beta sheets and primarily synthesized in the liver.[17, 18] In its soluble state, TTR is a tetrameric complex made up of four single chain monomers of TTR. The primary function of the TTR tetramer is to carry thyroxine and retinol (vitamin A)-retinol binding protein complex. The prevailing theory for the 
pathogenesis of TTR amyloidosis is that aging in ATTRwt or inherited mutations in ATTRm cause tetrameric TTR to dissociate into its monomeric subunits. These monomers misfold and aggregate into insoluble fibrillar structures that deposit in organs and tissues and eventually lead to organ failure. Distinct from AL amyloid where fibrils are toxic to myocardial cells, principally via the p38 MAPK signaling pathway[19], a toxicity of TTR has yet to be clearly elucidated.

Amyloid deposition in the myocardium resulting in heart failure is the leading cause of morbidity and mortality in patients with ATTR cardiac amyloidosis.[1, 8] Progressive left ventricular chamber dysfunction is mediated by physiologic derangements that include reduction in chamber capacitance, declines in chamber contractility and increases in arterial elastance, with resulting declines in the ventricle's ability to perform work.[20] ATTR cardiac amyloidosis has a similar echocardiographic appearance (Figure 1) as systemic lightchain cardiac amyloidosis (AL),[21] though wall thickness tends to be higher in ATTR than AL[22] despite a slower progression of disease in ATTR compared with AL. The worse survival in AL cardiac amyloid compared to ATTR cardiac amyloid has been attributed to the added toxicity of light chains to cardiomyocytes.[19, 23] Furthermore, ATTRwt cardiac amyloidosis appears to disproportionately afflict older adult men compared to women for reasons that have yet to be elucidated.

\section{CLINICAL FEATURES AND PROGNOSIS}

In the elderly patient with heart failure, several demographic, clinical, electrocardiographic (ECG), biomarker, and imaging characteristics should raise the suspicion for the diagnosis of cardiac amyloidosis, (Table 1), which has been underdiagnosed to date. First, demographically, ATTRwt cardiac amyloidosis predominately affects older men over 65 years of age.[6, 12, 13, 16, 21] While the vast majority of patients with ATTRwt and ATTRm Val122Ile had cardiac symptoms at presentation, those with ATTRwt had a higher incidence of rhythm disturbance (i.e. atrial fibrillation, sinoatrial block) than ATTRm due to the Val122Ile mutation (65.5\% vs 32\%). [16] ATTRwt is not typically associated with extracardiac organ involvement, although patients may present with a history of carpal tunnel syndrome or lumbar spinal stenosis that precedes a cardiac diagnosis by several years.[24, 25] Patients with ATTRm due to the Val122Ile mutation not only have a cardiac phenotype but may also have associated neuropathic and GI manifestations. In the Transthyretin Amyloid Outcomes Survey (THAOS), patients with ATTRm due to the Val122Ile mutation compared to ATTRwt had a higher incidence of gait instability (18.1\% vs. 6.7\%), gastrointestinal manifestations including diarrhea and/or constipation (18.9\% vs $8.6 \%$ ), neuropathic pain ( $33.8 \%$ vs. $12 \%)$, and urinary incontinence ( $4.1 \%$ vs. $0.6 \%)$, suggesting greater involvement of the peripheral and autonomic nervous system.[16] The third most common cause of ATTR cardiac amyloid in the United States reported in the THOAS registry is the Thr60Ala mutation (Appalachian mutation) which is associated with a greater degree of peripheral and autonomic involvement (Table 2)

Exercise intolerance is among the most common complaints expressed by patients with ATTR cardiac amyloidosis. Functional decline compounds risk associated with heart failure and results in diminished quality of life, increased frailty and higher mortality. 
Cardiopulmonary exercise testing has shown that maximal oxygen consumption $\left(\mathrm{VO}_{2} \mathrm{max}\right)$ was $13.5 \pm 4.9 \mathrm{~mL} \cdot \mathrm{kg}-1 \cdot \mathrm{min}-1$ in ATTRwt, which is $54 \%$ of age predicted. The ventilatory equivalent ratio for carbon dioxide $\left(\mathrm{Ve} / \mathrm{VCO}_{2}\right)$ slope, an index of ventilatory efficiency and predictor of mortality in heart failure, exceeded the upper limit of normal by $>35 \%$.[6] The reduced $\mathrm{VO}_{2}$ max is mediated by severe diastolic dysfunction with an inability to augment stroke work with exercise as well as by significant chronotropic incompetence.

Progressive cardiac conduction system disease and cardiac autonomic dysfunction are common in ATTR cardiac amyloidosis. While low electrocardiographic voltage is widely considered a diagnostic marker of cardiac amyloidosis and has been linked with worse survival, its overall sensitivity is less than $30 \%$ and it cannot differentiate between amyloid subtypes.[26, 27] The absence of low voltage, therefore, should not preclude the diagnosis of ATTR cardiac amyloid. Instead, a low voltage-to-mass ratio, which can be expressed in a relatively complex way (voltage measured by Sokolow criteria and left ventricular mass defined as cross-sectional area [28]) or by a simpler ratio (total QRS voltage [sum of QRS voltages in all the ECG leads] divided by indexed LV mass (g/m2)[29] have demonstrated a higher sensitivity for identifying cardiac amyloidosis.[26, 29] A pseudo infarct pattern with pathologic Q-waves is also more sensitive than low voltage in identifying cardiac amyloidosis.[26] Relative to patients with AL cardiac amyloidosis, those with ATTRwt have a higher incidence of atrial fibrillation and atrial flutter as well as symptomatic bradyarrhythmias that may progress to heart block, which are rhythm disturbances most likely attributable to their older age. Subsequently, compared to patients with AL cardiac amyloidosis, those with ATTRwt cardiac amyloidosis have a higher incidence of permanent pacemaker placement, which is associated with worse survival compared to patients with ATTRwt without a pacemaker.[12]

Cardiac biomarkers (natriuretic peptides and troponin) are useful for both the identification of ATTRwt and ATTRm in the geriatric patient and for risk stratification. While elevated Nterminal pro-B-type natriuretic peptide (NT pro-BNP) and persistent low level troponin elevations due to myocardial apoptosis are features of all subtypes of cardiac amyloidosis, elderly patients with ATTRwt typically have lower NT pro-BNP compared with patients with AL cardiac amyloidosis. In one study, patients older than 70 years with an NT pro-BNP $<1420 \mathrm{pmol} / \mathrm{L}$ were more likely to have ATTRwt relative to AL cardiac amyloidosis.[12] An elevated troponin in the absence of a clinical syndrome compatible with acute ischemia is often a clue to the presence of cardiac amyloidosis, as amyloid deposition in the myocardium causes apoptosis. In ATTRwt patients, using thresholds of troponin T $(0.05$ $\mathrm{ng} / \mathrm{ml})$ and NT-proBNP (3000 pg/ml), the respective 4-year overall survival estimates were $57 \%, 42 \%$, and $18 \%$ for stage I (both values below cutoff), stage II (one above) and Stage III (both above), respectively.[30]

\section{NON-BIOPSY DIAGNOSIS}

Recently, single and multicenter studies of bone isotope imaging using the technetium ( ${ }^{99 \mathrm{~m}} \mathrm{Tc}$ )-labeled bisphosphonates, pyrophosphate (PYP), 3,3-diphos-phono-1,2propanodicarboxylic acid (DPD), and hydroxymethylene diphosphonate (HMDP), have demonstrated excellent sensitivity and specificity for diagnosis of ATTR cardiac amyloidosis 
and differentiation from AL cardiac amyloidosis (Figure 1).[31-36] Specifically, the diagnosis of ATTR cardiac amyloidosis is suggested by a score of 2 or 3 using the Perugini visual score of myocardial radiotracer enhancement (range 0-3: score 0, absent cardiac uptake and normal bone uptake; score 1, mild cardiac uptake, inferior to bone uptake; score 2 , moderate cardiac uptake accompanied by attenuated bone uptake; score 3, strong cardiac uptake with mild/absent bone uptake). While endomyocardial biopsy and the use of mass spectrometry for tissue typing are considered the gold standard, in the context of advancements in diagnostic imaging, they may no longer be necessary.[35]

Using these imaging techniques, an algorithm for diagnosing elderly patients with ATTR cardiac amyloidosis has been proposed (Figure 2). An older adult with clinical, biomarker, ECG, echocardiographic, and/or cardiac magnetic resonance imaging suggestive of cardiac amyloidosis should first be offered patient-centered counseling about the risks and benefits of the diagnostic process that may lie ahead in order to advance their care. This may include counseling about further blood testing, nuclear imaging, genetic testing, and/or potential endomyocardial biopsy. Next, testing for AL cardiac amyloidosis with free light chain assay and serum/urine protein electrophoresis with immunofixation should be performed. Any patient with evidence of a monoclonal protein should proceed with tissue biopsy and typing, as AL amyloid can cause false positive myocardial uptake of technetium in some patients. A patient without evidence of a monoclonal protein may undergo bone isotope cardiac imaging, where diffuse myocardial enhancement and grade 2 or 3 uptake by Perugini score [31] is diagnostic of ATTR cardiac amyloidosis obviating the need for confirmatory tissue biopsy.[35] While the likelihood of hereditary ATTRm cardiac amyloidosis is low in an elderly white male, further genetic testing can differentiate ATTRm from ATTRwt cardiac amyloidosis, which has implications for genetic counseling of affected family members in patients screening positive for an inherited TTR mutation. Of note, there are a number of "non-pathologic" mutations, such as Gly6Ser, which are non-amyloidogenic. While bone isotope imaging can accurately differentiate ATTR from AL cardiac amyloidosis when there is no evidence of an abnormal monoclonal protein, it should be noted that up to $20 \%$ of patients with ATTRwt cardiac amyloidosis can also have a monoclonal protein, suggestive of an underlying plasma cell dyscrasia. In such patients, tissue biopsy with amyloid typing is required to definitively determine the type of cardiac amyloidosis (AL versus TTR).

\section{MANAGEMENT}

Heart failure in ATTR cardiac amyloid is managed with salt restriction and loop diuretics in combination with aldosterone antagonists. Often, loop diuretics with a higher potency or bioavailability (i.e. bumetanide or torsemide) are preferred, as many patients with ATTR cardiac amyloid have right sided heart failure which can result in gut edema and impair absorption of diuretics. For patients with advanced ATTRwt cardiac amyloidosis with hypotension and risk of syncope, midrodine may be employed. Angiotensin converting enzyme-inhibitors and angiotensin receptor blockers are often poorly tolerated due to concomitant autonomic dysfunction and risk of symptomatic hypotension.[3] High dose beta blockers are also poorly tolerated as the stroke volume in patients with cardiac amyloidosis is fixed and cardiac output is maintained by a physiologic increase in heart rate.[3] Nondihydropyridine calcium channel blockers are contraindicated as they can bind avidly to 
amyloid fibrils causing high-degree heart block and negative inotropic effects.[37, 38]

Similar concerns have been raised about digoxin because it can cause cardiac rhythm disturbances and sudden death.[39, 40] Paradoxically, permanent pacemaker placement has been associated with an increased risk of long term adverse outcomes,[12] but this may be related more to the fact that conduction disease alone is a marker of advanced disease than the pacemaker. Prospective data are lacking regarding the use of implantable cardioverterdefibrillators (ICD) in ATTR cardiac amyloidosis, but in general, despite having significant burden of ventricular arrhythmias on routine monitoring, these patients do not usually die suddenly from ventricular arrhythmias. Therefore, concerns have been raised about the use of ICDs in this population. Placement of an ICD lead can also cause or worsen tricuspid regurgitation which could exacerbate an already difficult to manage restrictive cardiomyopathy.

Orthotopic heart transplantation (OHT) has been performed in selected patients with ATTRwt and ATTRm Val122Ile cardiac amyloidosis with improving outcomes in the modern era. [41, 42] However, OHT is rarely feasible as most patients are diagnosed at an advanced age and with comorbid conditions which are contraindications to cardiac transplantation. Cardiac amyloid is considered a contraindication to placement of a left ventricular assist device as the small ventricular chamber size and increased wall thickness makes such patients prone to suction events and amyloid deposition in the right ventricle predisposes to right ventricular failure. Limited data on left ventricular assist devices have shown higher rates of complications compared to those with a dilated cardiomyopathy [43] and increased mortality in subjects with ventricular chamber size less than $46 \mathrm{~mm}$.[44]

There are emerging therapeutics that aim to inhibit synthesis of the TTR protein (silencers), stabilize serum TTR from breakdown into amyloidogenic monomers (stabilizers), or degrade and extract deposited amyloid fibrils (degraders and extractors). As outlined in Table 2, several compounds are in various phases of clinical development, but to date none of these agents are FDA approved for this condition. It is important to note that there has been a lack of animal models that recapitulate the human phenotype of TTR cardiac amyloid, which has placed more importance on non-invasive, repetitive imaging modality characterization of disease progression in a variety of clinical trials, as successful therapeutic application in a relevant animal model is limited.

TTR stabilizers have demonstrated efficacy in familial amyloid polyneuropathy (FAP)[45, 46], but their efficacy in ATTR cardiac amyloidosis is unknown. Diflunisal, FDA-approved as a non-steroidal anti-inflammatory drug, slowed neuropathy progression and improved quality of life in patients with FAP over 2 years but has risks of gastrointestinal bleeding, kidney injury, and heart failure exacerbation $[47,48]$ with conflicting studies regarding tolerance.[49, 50] Tafamidis is a TTR stabilizer that has no non-steroidal properties. In a phase 2 trial of subjects with senile cardiac amyloidosis, tafamidis effectively achieved and maintained TTR stabilization and was well tolerated. The absence of significant changes in most biochemical and echocardiographic parameters over a 12 month period was encouraging,[51,52] and has led to a phase 3 trial that has completed enrollment with results anticipated in 2018. 
Silencers consist of small ribonucleic acid (RNA) molecules that inhibit TTR protein synthesis.[53] Revusiran (ALN-TTRSc) is a specific small interfering RNA (siRNA) that is linked to the amino sugar galactose derivative, NAcetylgalactosamine (GalNAc) ligand. The GalNAc compound binds to the asialoglycoprotein receptor expressed on hepatocytes facilitating deliver of the siRNA to the liver which is the primary source of TTR production. Revusiran results in $>80 \%$ knock down of circulating TTR.[54] Revusiran, delivered via subcutaneous injection, was being evaluated in patients with ATTRm but not ATTRwt cardiac amyloidosis in a phase III trial called ENDEAVOUR and in a phase II trial that included both ATTRm and ATTRwt subjects. The sponsor recently halted development of revusiran because of new onset or worsening peripheral neuropathy in the phase II trial and because of an imbalance in mortality between revusiran and placebo in the phase III trial, ENDEAVOUR. Notably, the drug, patisiran, a lipid nanoparticle formulation of an siRNA to silence TTR that is administered intravenously is being tested in a phase III clinical trial, APOLLO. Another silencer, IONIS-TTR-RX, is an antisense oligonucleotide, which is a synthetic peptide that binds directly to TTR messenger RNA and leads to its degradation by the endonuclease, ribonuclease H.[55] It is currently being tested in a phase II open label trial and a phase III investigation for neuropathy in patients with FAP, with potential for a future phase III trial in ATTRwt cardiac amyloid.

A third strategy under study is TTR degradation and extraction. Doxycycline disrupts fibril formation and when combined in an animal model with the bile salt, tauroursodeoxycholic acid (TUDCA), demonstrated a synergistic effect in removing tissue deposits of TTR.[56] A corresponding open label human clinical showed that historical controls had a greater percent decline in myocardial strain than subjects treated with doxycycline and TUDCA,[57] and while BNP increased after 12 months, those receiving treatment had no change in troponin I, interventricular septal thickness, ejection fraction or $\mathrm{VO}_{2}$ max.[58] Another strategy employs a proline-derived small molecule, R-1-[6-[R-2-carboxy-pyrrolidin-1-yl]-6oxo-hexanoyl] pyrrolidine-2-carboxylic acid (CPHPC), that exploits the fact that all amyloid fibril deposits contain the non-fibrillar normal plasma protein, serum amyloid P component (SAP). CPHPC efficiently depletes SAP from the plasma but leaves some SAP in amyloid deposits, which can be removed when administering concomitant IgG anti-SAP antibodies, which incite an inflammatory multinucleated giant cell response. In a phase I trial, wholebody scans using iodinated SAP demonstrated depletion of hepatic amyloid in 50\% of patients with liver involvement. Although patients with cardiac involvement were excluded from this trial, there were also reductions in the inflammatory markers, complement component 3 and C-reactive protein.[59]

Finally, 2 additional agents have demonstrated therapeutic potential. The molecule, AG10, was synthesized as a TTR stabilizer designed to bind the inner cavity and periphery of the thyroxine 4 binding site. It was shown to stabilize wild type TTR better than tafamidis and diflunisal in in vitro studies.[60] Tolcapone, an FDA-approved anti-Parkinsonian drug, was also recently studied for its ability to stabilize TTR. In vitro, tolcapone was also shown to bind TTR better than tafamidis, and in vivo, it had a stabilizing effect.[61] 


\section{SUMMARY}

With the aging of the population, ATTRwt and ATTRm are likely to become the most common type of cardiac amyloidosis. ATTRwt and ATTRm cardiac amyloidosis can be diagnosed non-invasively (i.e. without histology) in patients with typical echocardiographic or cardiac MRI findings accompanied by grade 2 or 3 myocardial uptake on technetium pyrophosphate imaging and no evidence of a monoclonal protein. Morbidity and mortality from ATTRwt and ATTRm cardiac amyloidoses is high and the emergence of several therapies based on an understanding of the pathophysiology of this condition may provide alternate treatment strategies for those afflicted.

\section{REFERENCES}

1. Ruberg FL, Berk JL. Transthyretin (TTR) cardiac amyloidosis. Circulation. 2012; 126:1286-300. [PubMed: 22949539]

2. Westermark P. Aspects on human amyloid forms and their fibril polypeptides. The FEBS journal. 2005; 272:5942-9. [PubMed: 16302959]

3. Castano A, Drachman BM, Judge D, Maurer MS. Natural history and therapy of TTR-cardiac amyloidosis: emerging disease-modifying therapies from organ transplantation to stabilizer and silencer drugs. Heart failure reviews. 2015; 20:163-78. [PubMed: 25408161]

4. Pitkanen P, Westermark P, Cornwell GG 3rd. Senile systemic amyloidosis. The American journal of pathology. 1984; 117:391-9. [PubMed: 6507586]

5. Dharmarajan K, Maurer MS. Transthyretin cardiac amyloidoses in older North Americans. J Am Geriatr Soc. 2012; 60:765-74. [PubMed: 22329529]

6. Connors LH, Sam F, Skinner M, Salinaro F, Sun F, Ruberg FL, et al. Heart Failure Resulting From Age-Related Cardiac Amyloid Disease Associated With Wild-Type Transthyretin: A Prospective, Observational Cohort Study. Circulation. 2016; 133:282-90. [PubMed: 26660282]

7. Bureau UC. Current Estimates Data.

8. Wechalekar AD, Gillmore JD, Hawkins PN. Systemic amyloidosis. Lancet. 2016; 387:2641-54. [PubMed: 26719234]

9. Jacobson DR, Alexander AA, Tagoe C, Buxbaum JN. Prevalence of the amyloidogenic transthyretin (TTR) V122I allele in 14333 African-Americans. Amyloid. 2015; 22:171-4. [PubMed: 26123279]

10. Tanskanen M, Peuralinna T, Polvikoski T, Notkola IL, Sulkava R, Hardy J, et al. Senile systemic amyloidosis affects $25 \%$ of the very aged and associates with genetic variation in alpha2macroglobulin and tau: a population-based autopsy study. Ann Med. 2008; 40:232-9. [PubMed: 18382889]

11. Coelho T, Maurer MS, Suhr OB. THAOS - The Transthyretin Amyloidosis Outcomes Survey: initial report on clinical manifestations in patients with hereditary and wild-type transthyretin amyloidosis. Curr Med Res Opin. 2013; 29:63-76. [PubMed: 23193944]

12. Pinney JH, Whelan CJ, Petrie A, Dungu J, Banypersad SM, Sattianayagam P, et al. Senile systemic amyloidosis: clinical features at presentation and outcome. Journal of the American Heart Association. 2013; 2:e000098. [PubMed: 23608605]

13. Givens RC, Russo C, Green P, Maurer MS. Comparison of cardiac amyloidosis due to wild-type and V122I transthyretin in older adults referred to an academic medical center. Aging health. 2013; 9:229-35. [PubMed: 24073013]

14. González-López E, Gallego-Delgado M, Guzzo-Merello G, de Haro-Del Moral F, Cobo-Marcos $\mathrm{M}$, Robles $\mathrm{C}$, et al. Wild-type transthyretin amyloidosis as a cause of heart failure with preserved ejection fraction. Eur Heart J. 2015

15. Castano A, Bokhari S, Maurer MS. Unveiling wild-type transthyretin cardiac amyloidosis as a significant and potentially modifiable cause of heart failure with preserved ejection fraction. Eur Heart J. 2015 
16. Maurer MS, Hanna M, Grogan M, Dispenzieri A, Witteles R, Drachman B, et al. Genotype and Phenotype of Transthyretin Cardiac Amyloidosis: THAOS (Transthyretin Amyloid Outcome Survey). Journal of the American College of Cardiology. 2016; 68:161-72. [PubMed: 27386769]

17. Goodman, DS. Plasma retinol-binding protein. Academic Press; Orlando, FL.: 1984.

18. Buxbaum JN, Reixach N. Transthyretin: the servant of many masters. Cell Mol Life Sci. 2009; 66:3095-101. [PubMed: 19644733]

19. Liao R, Jain M, Teller P, Connors LH, Ngoy S, Skinner M, et al. Infusion of light chains from patients with cardiac amyloidosis causes diastolic dysfunction in isolated mouse hearts. Circulation. 2001; 104:1594-7. [PubMed: 11581134]

20. Bhuiyan T, Helmke S, Patel AR, Ruberg FL, Packman J, Cheung K, et al. Pressure-volume relationships in patients with transthyretin (ATTR) cardiac amyloidosis secondary to V122I mutations and wild-type transthyretin: Transthyretin Cardiac Amyloid Study (TRACS). Circulation Heart failure. 2011; 4:121-8. [PubMed: 21191093]

21. Ng B, Connors LH, Davidoff R, Skinner M, Falk RH. Senile systemic amyloidosis presenting with heart failure: a comparison with light chain-associated amyloidosis. Archives of internal medicine. 2005; 165:1425-9. [PubMed: 15983293]

22. Quarta CC, Solomon SD, Uraizee I, Kruger J, Longhi S, Ferlito M, et al. Left ventricular structure and function in transthyretin-related versus light-chain cardiac amyloidosis. Circulation. 2014; 129:1840-9. [PubMed: 24563469]

23. Mishra S, Guan J, Plovie E, Seldin DC, Connors LH, Merlini G, et al. Human amyloidogenic light chain proteins result in cardiac dysfunction, cell death, and early mortality in zebrafish. Am J Physiol Heart Circ Physiol. 2013; 305:H95-103. [PubMed: 23624626]

24. Sekijima Y, Uchiyama S, Tojo K, Sano K, Shimizu Y, Imaeda T, et al. High prevalence of wildtype transthyretin deposition in patients with idiopathic carpal tunnel syndrome: a common cause of carpal tunnel syndrome in the elderly. Hum Pathol. 2011; 42:1785-91. [PubMed: 21733562]

25. Westermark P, Westermark GT, Suhr OB, Berg S. Transthyretin-derived amyloidosis: probably a common cause of lumbar spinal stenosis. Ups J Med Sci. 2014; 119:223-8. [PubMed: 24620715]

26. Cyrille NB, Goldsmith J, Alvarez J, Maurer MS. Prevalence and prognostic significance of low QRS voltage among the three main types of cardiac amyloidosis. Am J Cardiol. 2014; 114:108993. [PubMed: 25212550]

27. Sperry BW, Vranian MN, Hachamovitch R, Joshi H, Ikram A, Phelan D, et al. Subtype-Specific Interactions and Prognosis in Cardiac Amyloidosis. J Am Heart Assoc. 2016; 5:e002877. [PubMed: 27013539]

28. Carroll JD, Gaasch WH, McAdam KP. Amyloid cardiomyopathy: characterization by a distinctive voltage/mass relation. Am J Cardiol. 1982; 49:9-13. [PubMed: 6459025]

29. Quarta CC PS, Longhi S, Berardini A, Musca F, Salinaro F, Obici L, Milandri A, Gallo P, Gagliardi P, Biagini E, Mingardi F, Pazzi C, Merlini G, Rapezzi C. A Simple Voltage/Mass Index Improved Diagnsois of Cardiac Amyloidosis; An Electrocardiographic and Echocardiographic Study of 570 Patients with Left Ventricular Hypertrophy. JACC. 2012; 59:E1586-E.

30. Grogan M SC, Kyle RA, Zeldenrust SR, Gertz MA, Lin G, Klarich KW, Miller WL, Maleszewski JJ, Dispenzieri A. Natural History of Wild Type Transthyretin Cardiac Amyloidosis and Risk Stratification Using a Novel Staging System. JACC. 2016; 68:1014-20. [PubMed: 27585505]

31. Rapezzi C, Guidalotti P, Salvi F, Riva L, Perugini E. Usefulness of 99mTc-DPD scintigraphy in cardiac amyloidosis. J Am Coll Cardiol. 2008; 51:1509-10. author reply 10. [PubMed: 18402909]

32. Hutt DF, Quigley AM, Page J, Hall ML, Burniston M, Gopaul D, et al. Utility and limitations of 3,3-diphosphono-1,2-propanodicarboxylic acid scintigraphy in systemic amyloidosis. Eur Heart J Cardiovasc Imaging. 2014; 15:1289-98. [PubMed: 24939945]

33. Galat A, Rosso J, Guellich A, Van Der Gucht A, Rappeneau S, Bodez D, et al. Usefulness of $(99 \mathrm{~m}) \mathrm{Tc}-\mathrm{HMDP}$ scintigraphy for the etiologic diagnosis and prognosis of cardiac amyloidosis. Amyloid. 2015; 22:210-20. [PubMed: 26465835]

34. Bokhari S, Castano A, Pozniakoff T, Deslisle S, Latif F, Maurer MS. (99m)Tc-pyrophosphate scintigraphy for differentiating light-chain cardiac amyloidosis from the transthyretin-related familial and senile cardiac amyloidoses. Circ Cardiovasc Imaging. 2013; 6:195-201. [PubMed: 23400849] 
35. Gillmore JD, Maurer MS, Falk RH, Merlini G, Damy T, Dispenzieri A, et al. Nonbiopsy Diagnosis of Cardiac Transthyretin Amyloidosis. Circulation. 2016; 133:2404-12. [PubMed: 27143678]

36. Castano A HM, Narotsky D, Goldsmith J, Weinberg RL, Morgenstern R, Pozniakoff T, Ruberg FL, Miller EJ, Berk JL, Dispenzieri A, Grogan M, Johnson G, Bokhari S, Maurer MS. Multicenter Experience of Planar Technetium 99m Pyrophosphate Cardiac Imaging: Predicting Survival for Patients with ATTR Cardiac Amyloidosis. JAMA Cardiology. 2016

37. Gertz MA, Falk RH, Skinner M, Cohen AS, Kyle RA. Worsening of congestive heart failure in amyloid heart disease treated by calcium channel-blocking agents. Am J Cardiol. 1985; 55:1645. [PubMed: 4003314]

38. Pollak A, Falk RH. Left ventricular systolic dysfunction precipitated by verapamil in cardiac amyloidosis. Chest. 1993; 104:618-20. [PubMed: 8339658]

39. Cassidy JT. Cardiac amyloidosis. Two cases with digitalis sensitivity. Ann Intern Med. 1961; 55:989-94. [PubMed: 13877133]

40. Pomerance A. Senile cardiac amyloidosis. British heart journal. 1965; 27:711-8. [PubMed: 5829755]

41. Davis MK, Kale P, Liedtke M, Schrier S, Arai S, Wheeler M, et al. Outcomes after heart transplantation for amyloid cardiomyopathy in the modern era. Am J Transplant. 2015; 15:650-8. [PubMed: 25648766]

42. Davis MK, Lee PH, Witteles RM. Changing outcomes after heart transplantation in patients with amyloid cardiomyopathy. J Heart Lung Transplant. 2015; 34:658-66. [PubMed: 25444369]

43. Topilsky Y, Pereira NL, Shah DK, Boilson B, Schirger JA, Kushwaha SS, et al. Left ventricular assist device therapy in patients with restrictive and hypertrophic cardiomyopathy. Circ Heart Fail. 2011; 4:266-75. [PubMed: 21303989]

44. Grupper A, Park SJ, Pereira NL, Schettle SD, Gerber Y, Topilsky Y, et al. Role of ventricular assist therapy for patients with heart failure and restrictive physiology: Improving outcomes for a lethal disease. J Heart Lung Transplant. 2015; 34:1042-9. [PubMed: 25940074]

45. Berk JL, Suhr OB, Obici L, Sekijima Y, Zeldenrust SR, Yamashita T, et al. Repurposing diflunisal for familial amyloid polyneuropathy: a randomized clinical trial. Jama. 2013; 310:2658-67. [PubMed: 24368466]

46. Coelho T, Maia LF, Martins da Silva A, Waddington Cruz M, Plante-Bordeneuve V, Lozeron P, et al. Tafamidis for transthyretin familial amyloid polyneuropathy: a randomized, controlled trial. Neurology. 2012; 79:785-92. [PubMed: 22843282]

47. Epstein M. Non-steroidal anti-inflammatory drugs and the continuum of renal dysfunction. $\mathrm{J}$ Hypertens Suppl. 2002; 20:S17-23.

48. Mukherjee D, Nissen SE, Topol EJ. Risk of cardiovascular events associated with selective COX-2 inhibitors. JAMA. 2001; 286:954-9. [PubMed: 11509060]

49. Castano A, Helmke S, Alvarez J, Delisle S, Maurer MS. Diflunisal for ATTR cardiac amyloidosis. Congest Heart Fail. 2012; 18:315-9. [PubMed: 22747647]

50. Sekijima Y, Dendle MA, Kelly JW. Orally administered diflunisal stabilizes transthyretin against dissociation required for amyloidogenesis. Amyloid. 2006; 13:236-49. [PubMed: 17107884]

51. Merlini G, Plante-Bordeneuve V, Judge DP, Schmidt H, Obici L, Perlini S, et al. Effects of tafamidis on transthyretin stabilization and clinical outcomes in patients with non-Val30Met transthyretin amyloidosis. Journal of cardiovascular translational research. 2013; 6:1011-20. [PubMed: 24101373]

52. Maurer MS, Grogan DR, Judge DP, Mundayat R, Packman J, Lombardo I, et al. Tafamidis in transthyretin amyloid cardiomyopathy: effects on transthyretin stabilization and clinical outcomes. Circ Heart Fail. 2015; 8:519-26. [PubMed: 25872787]

53. Kanasty R, Dorkin JR, Vegas A, Anderson D. Delivery materials for siRNA therapeutics. Nat Mater. 2013; 12:967-77. [PubMed: 24150415]

54. Hawkins PN, Ando Y, Dispenzeri A, Gonzalez-Duarte A, Adams D, Suhr OB. Evolving landscape in the management of transthyretin amyloidosis. Ann Med. 2015; 47:625-38. [PubMed: 26611723] 
55. Ackermann EJ, Guo S, Booten S, Alvarado L, Benson M, Hughes S, et al. Clinical development of an antisense therapy for the treatment of transthyretin-associated polyneuropathy. Amyloid. 2012; 19(Suppl 1):43-4. [PubMed: 22494066]

56. Cardoso I, Martins D, Ribeiro T, Merlini G, Saraiva MJ. Synergy of combined doxycycline/ TUDCA treatment in lowering Transthyretin deposition and associated biomarkers: studies in FAP mouse models. J Transl Med. 2010; 8:74. [PubMed: 20673327]

57. Mirto T, Dupee D, Geller H, Falk RH. Doxycycline and TUDCA combination slow the progression of ATTR amyloid cardiomyopathy in humans. The XVth International Symposium on Amyloidosis. 2016:PA91.

58. Berk J, Ruberg FL, Pawar S, Brueckner CA, Wiesman JF, Stern L, Havasi A, Ward JE, Sanchorawala V. Doxycycline treatment of amyloidosis: a phase II study. The XVth International Symposium on Amyloidosis. Uppsala, Sweden. 2016:PA110.

59. Richards DB, Cookson LM, Berges AC, Barton SV, Lane T, Ritter JM, et al. Therapeutic Clearance of Amyloid by Antibodies to Serum Amyloid P Component. The New England journal of medicine. 2015; 373:1106-14. [PubMed: 26176329]

60. Penchala SC, Connelly S, Wang Y, Park MS, Zhao L, Baranczak A, et al. AG10 inhibits amyloidogenesis and cellular toxicity of the familial amyloid cardiomyopathy-associated V122I transthyretin. Proc Natl Acad Sci U S A. 2013; 110:9992-7. [PubMed: 23716704]

61. Sant'Anna R, Gallego P, Robinson LZ, Pereira-Henriques A, Ferreira N, Pinheiro F, et al. Repositioning tolcapone as a potent inhibitor of transthyretin amyloidogenesis and associated cellular toxicity. Nature communications. 2016; 7:10787. 


\section{Highlights}

- $\quad$ TTR cardiac amyloidosis is the quintessential form of diastolic heart failure that almost exclusively affects older adults

- $\quad$ As the population ages, the diagnosis of ATTRwt will increase making it the most common form of cardiac amyloidosis.

- $\quad$ A high index of suspicion is critical for making the diagnosis of cardiac amyloidosis.

- $\quad$ Nuclear scintigraphy agents using ${ }^{99 \mathrm{~m}} \mathrm{Tc}$-phosphate derivatives combined with assessment for monoclonal proteins are eliminating the need for tissue confirmation in ATTR cardiac amyloid. 

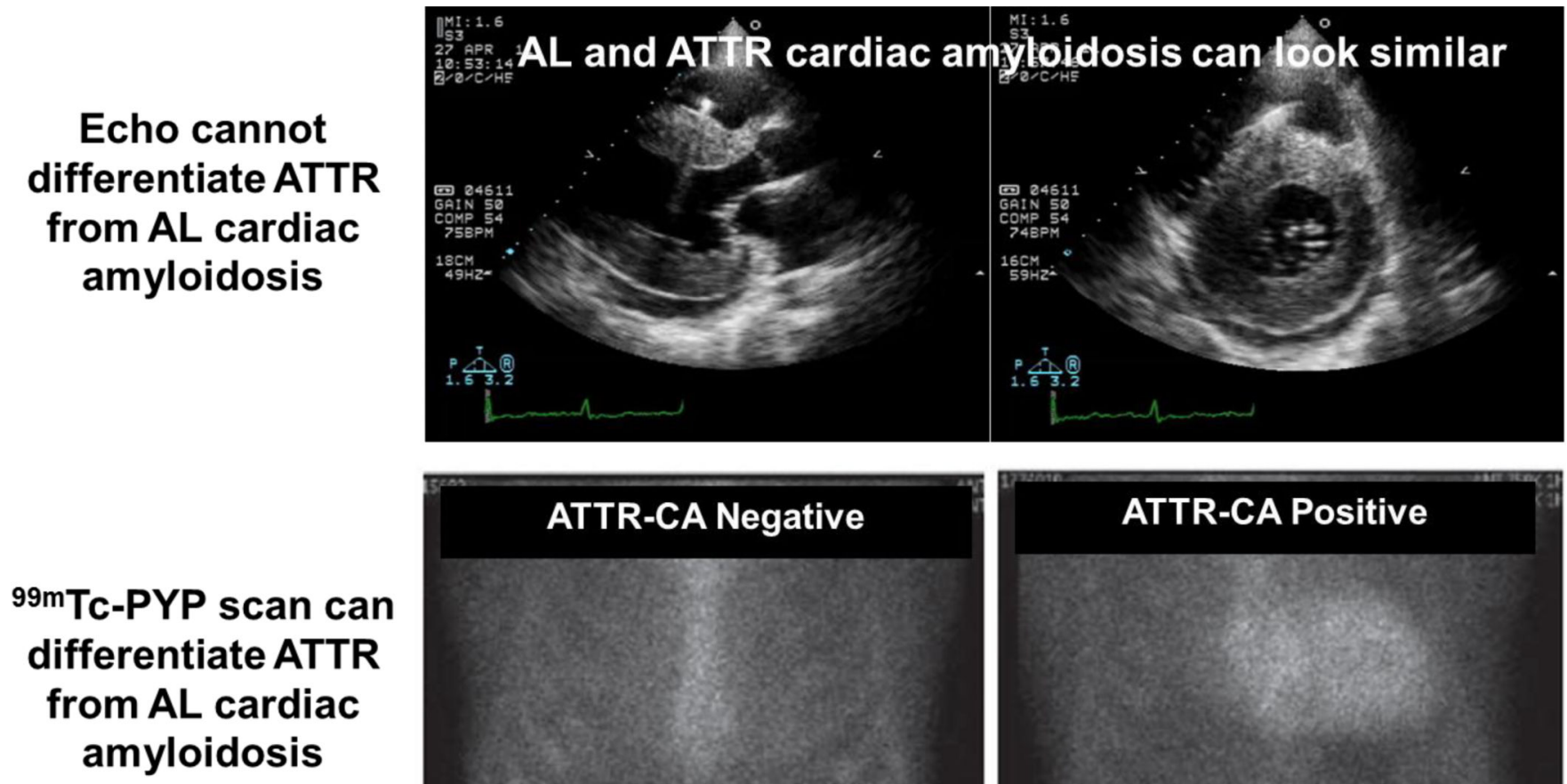

99mTc-PYP scan can differentiate ATTR from $A L$ cardiac amyloidosis

ATTR-CA Negative

\section{Raw Image}

\section{ATTR-CA Positive}

Figure 1.

Imaging in ATTR and AL Cardiac Amyloidosis 


\section{Heightened Clinical Suspicion for Cardiac Amyloid}

Older adult with clinical, biomarker, ECG, echocardiogram, and/or MRI imaging suggestive of cardiac amyloidosis

\section{Diagnostic Counseling \\ Patient-centered counseling on diagnostic process which may include further blood testing, nuclear imaging, genetic testing, and potential endomyocardial biopsy}

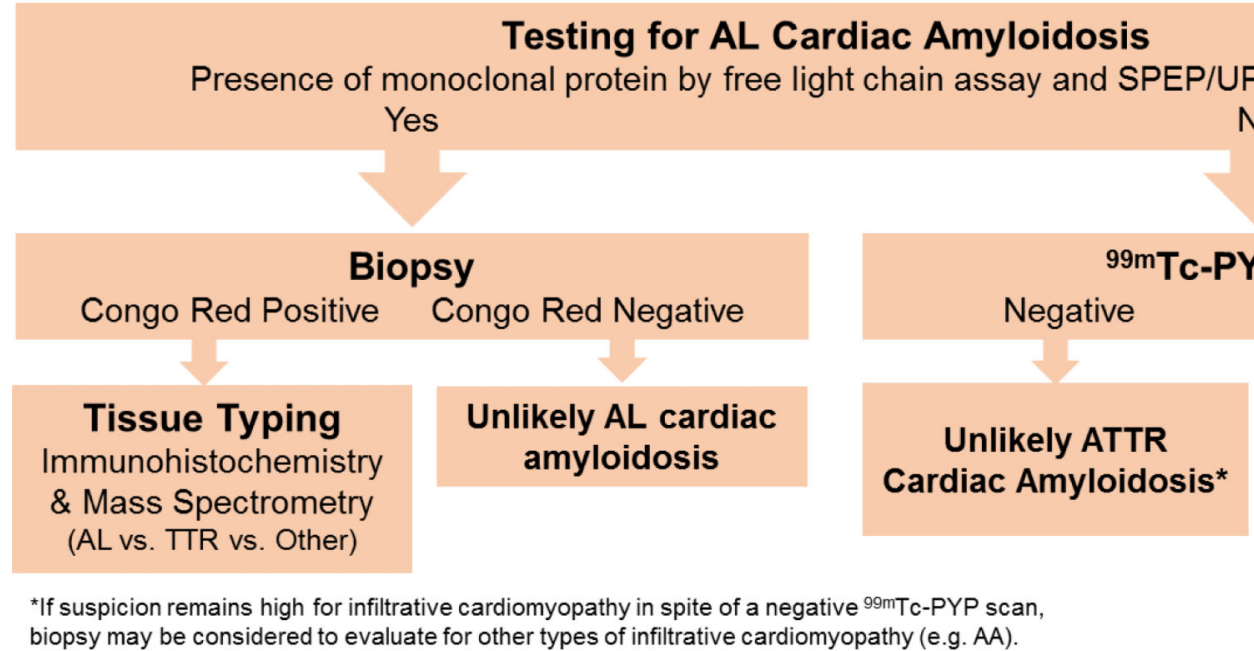

No

Abbreviations: ATTR, transthyretin cardiac amyloidosis; ATTRm, mutant transthyretin cardiac amyloidosis; ATTRwt, wild type transthyretin cardiac amyloidosis; ECG, electrocardiogram; IFE, immunofixation; MRI, magnetic resonance imaging; SPEP, serum protein electrophoresis; UPEP, urine protein electrophoresis.

Figure 2.

Proposed Diagnostic Algorithm for Older Patients with Suspected TTR Cardiac

Amyloidosis 


\section{Table 1}

Features that Should Elevate Suspicion for Cardiac Amyloidosis

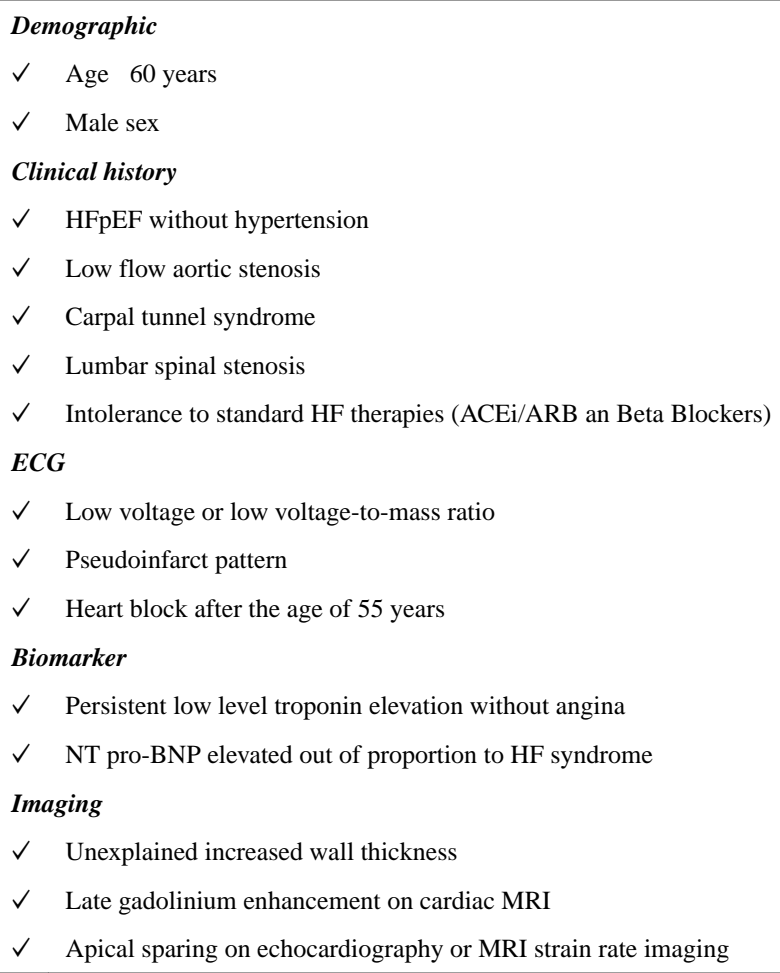

Abbreviations: ACEi, angiotensin converting enzyme inhibitor; ARB, angiotensin receptor blocker; HF, heart failure; HFpEF, heart failure with preserved ejection fraction; NT pro-BNP, amino-terminal pro-B-type natriuretic peptide; MRI, magnetic resonance imaging. 
Table 2

Comparison of Most Common Type of ATTR Cardiac Amyloidosis in Americans

\begin{tabular}{lccc}
\hline & ATTRwt & Val1221le & Thr60Ala \\
\hline Age & & & \\
Average & 75 & 70 & 60 \\
Age Range & 65 and up & 60 and up & 45 and up \\
Gender & $>90 \%$ Males reported to date & Male predominance & Equal \\
Ethnicity & Caucasian & Afro-Carribbean & Irish \\
Peripheral Neuropathy & + & ++ & +++ \\
Autonomic Neuropathy & + & ++ & +++ \\
\hline
\end{tabular}




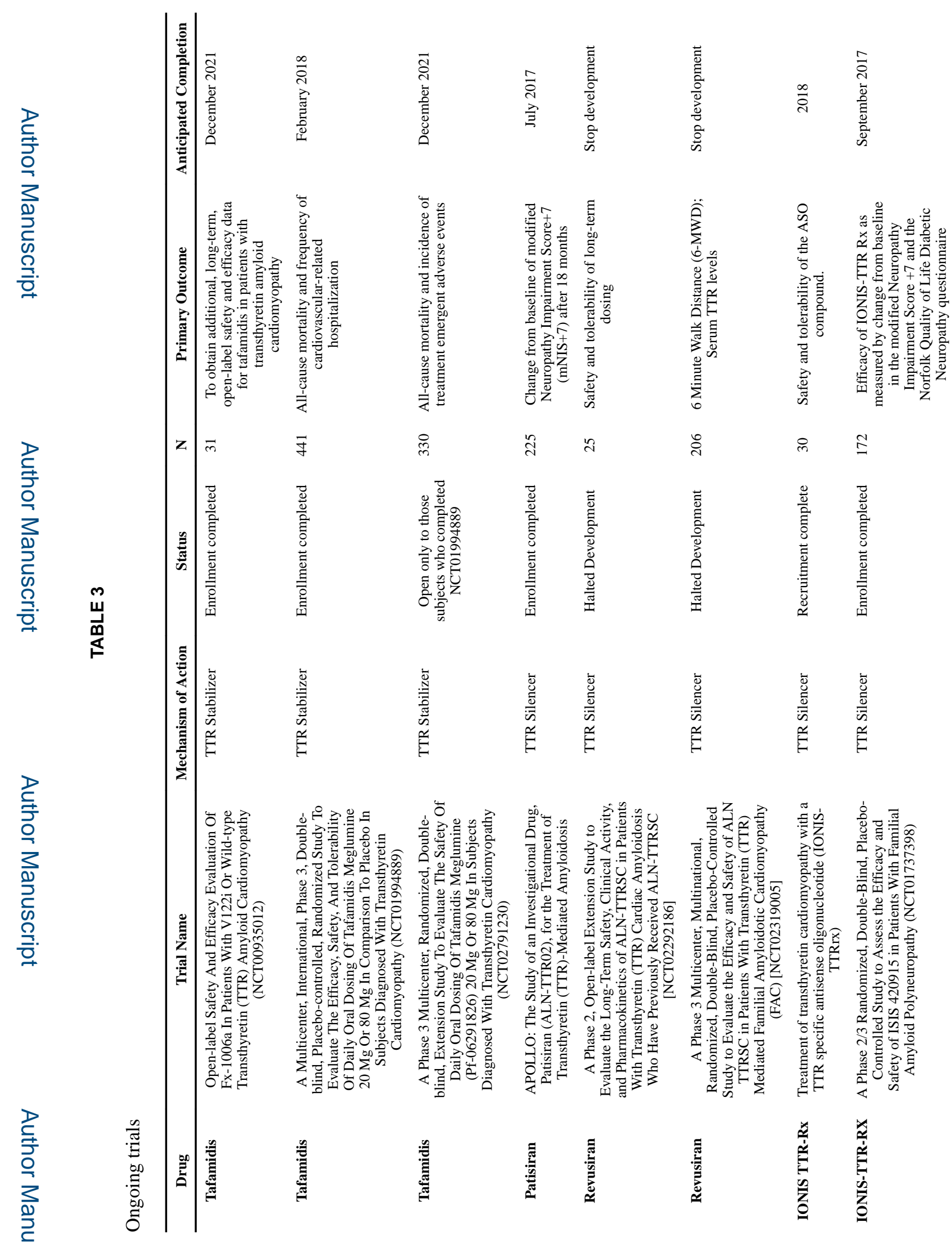

JCard Fail. Author manuscript; available in PMC 2017 December 01. 
Brunjes et al.

Page 18

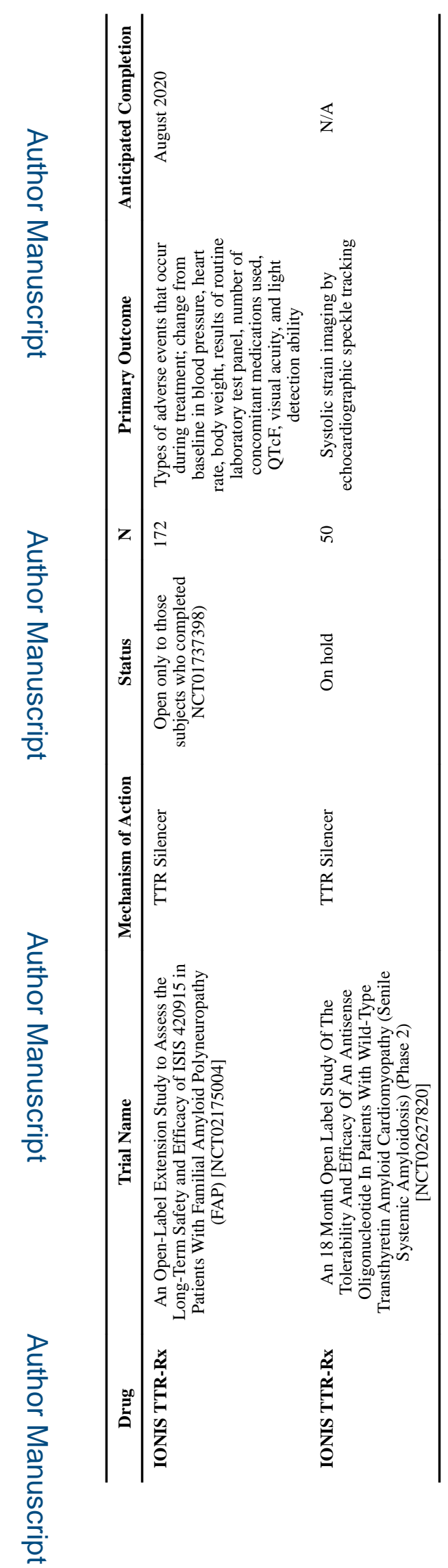

J Card Fail. Author manuscript; available in PMC 2017 December 01. 